\title{
Charge-Carrier Trapping and Radiative Recombination in Metal Halide Perovskite Semiconductors
}

Michael J. Trimpl, Adam D. Wright, Kelly Schutt, Leonardo R. V. Buizza, Zhiping Wang, Michael B. Johnston, Henry J. Snaith, Peter Müller-Buschbaum, Laura M. Herz*

Michael J. Trimpl, Dr. Adam D. Wright, Kelly Schutt, Leonardo R. V. Buizza, Dr. Zhiping Wang, Prof. Dr. Michael B. Johnston, Prof. Dr. Henry J. Snaith, Prof. Dr. Laura M. Herz Department of Physics, University of Oxford, Clarendon Laboratory, Parks Road, Oxford OX1 3PU, United Kingdom

E-mail: laura.herz@physics.ox.ac.uk

Michael Johann Trimpl, Prof. Dr. Peter Müller-Buschbaum

Technische Universität München, Physik Department, Lehrstuhl für Funktionelle Materialien, James-Franck-Str. 1, 85748 Garching, Germany

Prof. P. Müller-Buschbaum

Heinz Maier-Leibnitz Zentrum, Lichtenbergstr. 1, 85748 Garching, Germany

\section{Keywords:}

perovskite, trapping, photoluminescence, trap states, charge-carrier accumulation

\section{Abstract:}

Trap-related charge-carrier recombination fundamentally limits the performance of perovskite solar cells and other optoelectronic devices. While improved fabrication and passivation techniques have reduced trap densities, the properties of trap states and their impact on the charge-carrier dynamics in metal-halide perovskites are still under debate. Here, a unified model is presented of the radiative and non-radiative recombination channels in a mixed formamidinium-cesium lead iodide perovskite, including charge-carrier trapping, de-trapping and accumulation, as well as higher-order recombination mechanisms. A fast initial photoluminescence (PL) decay component observed after pulsed photogeneration is demonstrated to result from rapid localization of free charge carriers in unoccupied trap states, which may be followed by de-trapping, or non-radiative recombination with free carriers of opposite charge. Such initial decay components are shown to be highly sensitive to remnant charge-carriers that accumulate in traps under pulsed-laser excitation, with partial trap occupation masking the trap density actually present in the material. Finally, such modelling 


\section{WILEY-VCH}

reveals a change in trap density at the phase transition, and disentangles the radiative and nonradiative charge recombination channels present in $\mathrm{FA}_{0.95} \mathrm{Cs}_{0.05} \mathrm{PbI}_{3}$, accurately predicting the experimentally recorded PL efficiencies between 50 and $295 \mathrm{~K}$, and demonstrating that bimolecular recombination is a fully radiative process.

\section{Introduction}

Metal halide perovskites (MHPs) have attracted widespread attention in recent years because of their excellent optoelectronic properties, including long charge-carrier lifetimes, high charge-carrier mobilities and high absorption coefficients in the visible spectrum. ${ }^{[1]}$ These properties have enabled the recent success of MHPs in solar cell applications, with singlejunction cells now reaching efficiencies in excess of $25 \%,{ }^{[2]}$ rivalling those of commercial silicon solar cells. In contrast to silicon cells, MHPs offer a large variety of facile processing routes based e.g. on solution precursors or vapor deposition. ${ }^{[3]}$ However, current fabrication methods still result in a low but appreciable density of defect states in the perovskite structure that cause non-radiative recombination of charge-carriers. ${ }^{[4]}$ Trap-mediated recombination fundamentally hinders solar cells from reaching their highest possible efficiencies (the Shockley-Queisser limit ${ }^{[5]}$ ) at which charge-carrier extraction is exclusively limited by bandto-band radiative recombination of electrons with holes. ${ }^{[6]}$ Therefore, a reduction in trapmediated recombination is a prerequisite to reaching high efficiency devices, and is reflected in high photoluminescence quantum efficiencies (PLQE) close to unity, for which radiative bimolecular band-to-band recombination dominates over non-radiative pathways.

Much progress has recently been made in reducing the trap densities in MHPs, and in particular in passivating surface defects, ${ }^{[7]}$ with some surface treatments now resulting in high PLQEs of over $90 \%$ for $\mathrm{MAPbI}_{3}\left(\mathrm{MA} \equiv \mathrm{CH}_{3} \mathrm{NH}_{3}\right) .{ }^{[8]}$ However, despite recent advances in controlling perovskite film formation and trap passivation, and in understanding the key role 


\section{WILEY-VCH}

they play for reaching the radiative limit, the origin and properties of traps in MHPs are still poorly understood. ${ }^{[3 c, 8]}$ In particular, while trap states in MHPs may derive from point defects of the lattice, such as atomic vacancies, interstitials or substitutions, ${ }^{[9]}$ their precise specifications and nature are still the subject of intense debate. The relative energies of these defect states with respect to the conduction and valence band (the trap depths) have been inferred from both experimental and theoretical approaches, including photoluminescence (PL) transient measurements and density functional theory calculations. ${ }^{[4 c, 10]}$ As a result, various trap depths in methylammonium lead triiodide have been reported, ranging from $10 \mathrm{meV}$ up to $200 \mathrm{meV} .^{[11]}$ In addition, theoretical analysis of trap states has shown that the prominence of shallow traps and small phonon energies in MHP impedes non-radiative recombination and leads to slow charge-carrier decays, ${ }^{[12]}$ which may be one reason for the long PL lifetimes observed in these materials. ${ }^{[13]}$ Given that traps heavily influence the efficiencies of MHP devices, a unified picture of trap-mediated recombination, its impact on charge-carrier dynamics and the resulting PLQE of a perovskite is highly desirable. Such knowledge of the charge-carrier trapping kinetics and properties of trap states can ultimately be used to design materials with reduced trap densities and depths, and hence, lead to highperforming solar cell devices.

In this study, we investigate the dynamics of charge-carriers in a lead iodide perovskite across a range of excitation fluences and temperatures. To describe the observed PL transients, we develop a model that accounts for charge-carrier trapping, de-trapping and accumulation, as well as higher-order recombination mechanisms. Through our combined experimental and modelling approach, we are able to reveal the impact of temperature and phase transitions on the density of trap states present in the material, as well as the relative contributions arising from trap-mediated, band-to-band and Auger recombination. We focus on low to intermediate initial photoexcited charge-carrier densities around $10^{14}-10^{16} \mathrm{~cm}^{-3}$, in order to capture the 


\section{WILEY-VCH}

range encompassing AM1.5 solar illumination conditions. We link a fast initial

photoluminescence (PL) decay component observed after pulsed photogeneration to the rapid localization of free charge carriers in unoccupied trap states and demonstrate that these components depend sensitively on the repetition rate of the excitation pulse train. A fraction of charge-carriers created by previous pulses clearly remains in trap states prior to the arrival of the next excitation pulse, masking the density of traps actually present in the material. In addition, we use our model to unravel the radiative and non-radiative contributions to the overall PL transients, from which we are able to predict an expected photoluminescence quantum efficiency (PLQE). Good agreement with the actually measured PLQE reveals that all radiative emission derives from bimolecular band-recombination of electrons and holes. Ultimately, this work presents a unified view of the radiative and non-radiative charge-carrier recombination channels in a prototypical lead halide perovskite.

\section{Results and Discussion}

\subsection{Temperature-Dependent Charge-Carrier Dynamics}

We commence our investigation by experimentally assessing the dynamics of charge carriers through PL transient spectroscopy for a range of excitation fluences and sample temperatures. Full experimental details of the time-integrated and transient PL measurements are provided in Sections 4 and 6 of the Supporting Information, respectively. We chose $\mathrm{FA}_{0.95} \mathrm{Cs}_{0.05} \mathrm{PbI}_{3}$ $\left(\mathrm{FA} \equiv \mathrm{CH}\left(\mathrm{NH}_{2}\right)_{2}\right)$ thin films as a prototypical MHP for our investigation, which combines thermal stability (through absence of MA) with high crystalline quality (through incorporation of small fractions of Cs). ${ }^{[14]}$ To ensure that these results are generally representative for the group of similar materials, we compared data to those recorded for $\mathrm{FA}_{0.83} \mathrm{Cs}_{0.17} \mathrm{PbI}_{3}$ thin films that had been prepared by different researchers and slightly different methods. Full details of preparation methods for both materials are provided in Section 1 of the Supporting 


\section{WILEY-VCH}

Information. As shown in Section 6 of the Supporting Information, the observed PL dynamics for both samples are very similar in their nature and temperature dependence.

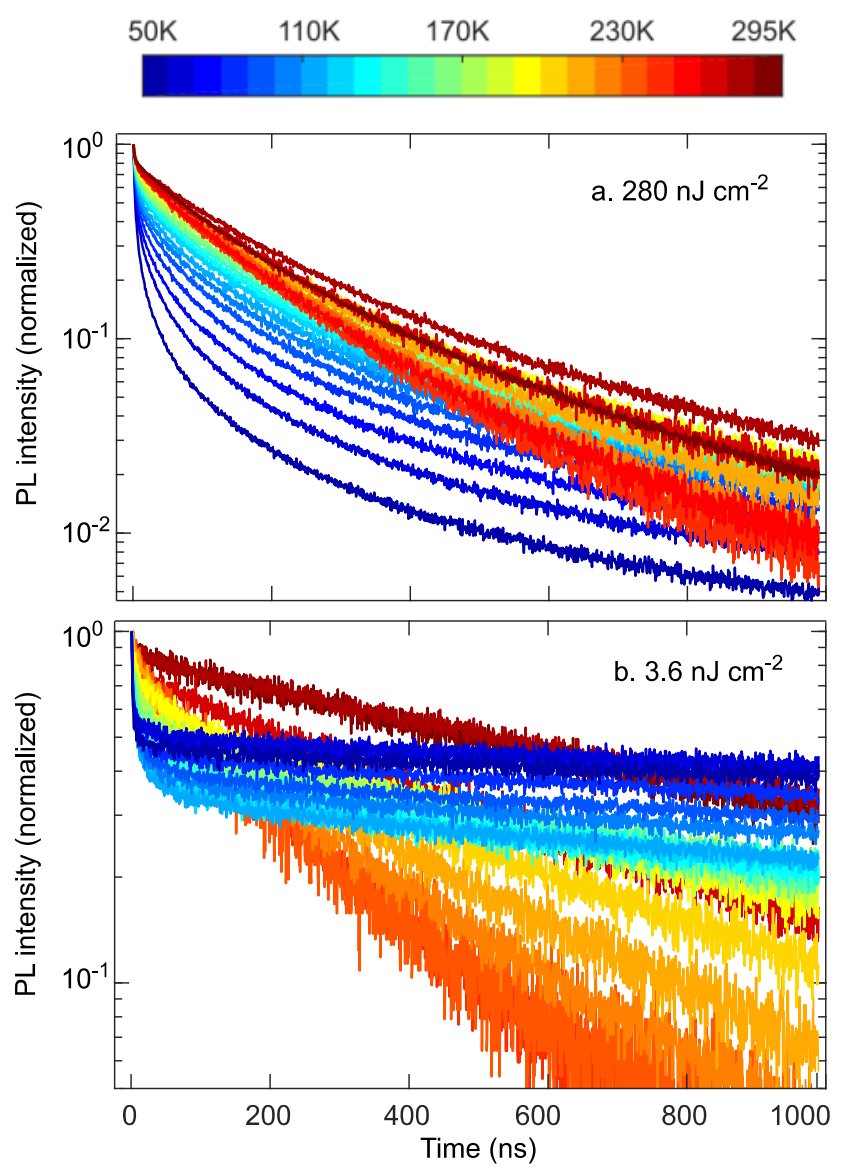

Figure 1. Normalized PL transients recorded for a $\mathrm{FA}_{0.95} \mathrm{Cs}_{0.05} \mathrm{PbI}_{3}$ thin film at two excitation fluences across temperatures ranging from $50 \mathrm{~K}$ to $295 \mathrm{~K}$. PL transients are detected at the PL emission peak at each temperature, ranging from $\lambda_{\mathrm{PL}}=812 \mathrm{~nm}$ to $\lambda_{\mathrm{PL}}=850 \mathrm{~nm}$ at a photoexcitation wavelength of $398 \mathrm{~nm}$. Bimolecular recombination dominates at higher fluences and increases with decreasing temperature. Trap-mediated recombination increases towards higher temperature and is most prominent in the low fluence regime.

We first examine the changes observed in the PL transients with excitation fluence and temperature in order to form a qualitative picture of the contributing charge-carrier recombination mechanisms. Further below, we subsequently describe a quantitative model that replicates these data and allows us to discern radiative and non-radiative contributions to the overall PL emission and radiative efficiency, while also providing insight into the trap state properties in these materials. 


\section{WILEY-VCH}

Figure 1 displays PL transient measurements for temperatures ranging from $50 \mathrm{~K}$ to $295 \mathrm{~K}$, at high and low excitation fluences. Qualitatively, the observed changes can be explained by the interplay between different charge-carrier recombination channels, which include trapmediated, band-to-band (bimolecular) and third-order Auger recombination. For the scenario in which trap filling and saturation are relatively unimportant to the overall carrier dynamics (e.g. at elevated excitation fluences), these three recombination mechanisms can be approximately captured by the following rate equation ${ }^{[1 \mathrm{a}, 1 \mathrm{~b}]}$

$\frac{\mathrm{d} n}{\mathrm{~d} t}=-R_{\mathrm{SRH}} n-R_{\mathrm{eh}} n^{2}-R_{\text {Auger }} n^{3}$

Here, trap-mediated monomolecular recombination described by the rate constant $R_{\mathrm{SRH}}$, has been previously attributed to Shockley-Read-Hall (SRH) recombination and is typically considered to be a non-radiative process. Electrons or holes are trapped in an intermediate trap state and ultimately non-radiatively recombine with holes or electrons, respectively. ${ }^{[5]}$ Bimolecular recombination, captured by the rate constant $R_{\text {eh, }}$, results from radiative band-toband recombination of free electrons in the conduction band with free holes in the valence band. ${ }^{[6]}$ Finally, Auger recombination, described by $R_{\text {Auger, }}$ is a third-order process that leads to recombination of an electron with a hole and requires energy and momentum transfer to an additional electron or hole. As Auger recombination involves three charge carriers, it is highly dependent on the charge-carrier density and only begins to contribute significantly at high charge-carrier densities $\left(\approx 10^{16} \mathrm{~cm}^{-3}-10^{17} \mathrm{~cm}^{-3}\right)$, and becomes dominant for $n>R_{\text {eh }} / R_{\text {Auger }} .^{[1 \mathrm{~b}]}$

The trends in PL dynamics displayed in Figure 1 qualitatively reflect the differences in the temperature dependencies of the three recombination pathways. At the lowest fluence $\left(3.6 \mathrm{~nJ} \mathrm{~cm}{ }^{-2}\right)$, the PL transients follow a monoexponential decay at longer times after 


\section{WILEY-VCH}

excitation, which suggests that trap-mediated recombination is dominating the charge-carrier dynamics and higher-order recombination mechanisms are negligible. Visual inspection shows that the rate of such trap-mediated charge-carrier recombination generally increases with increasing temperature. This observation is in agreement with the temperature dependence expected from SRH recombination, as previously observed in silicon and other materials. ${ }^{[16]}$ We note however that measurements above $250 \mathrm{~K}$ revealed a slight lowering of rates towards higher temperatures which may be related to a potential phase transition ${ }^{[17]}$ that can alter charge-carrier recombination rates. ${ }^{[18]}$

For higher excitation fluence $\left(280 \mathrm{~nJ} \mathrm{~cm}^{-2}\right)$ bimolecular band-to-band recombination is expected to dominate the PL dynamics. Here, we find that charge-carrier recombination rates instead decrease with increasing temperature. These trends are consistent with previous reports that have suggested a decrease in bimolecular band-to-band recombination with increasing temperatures, ${ }^{[11 \mathrm{a}]}$ because of a broadening of the Fermi-Dirac distribution functions, ${ }^{[6]}$ which results in more thermal spreading of electrons and holes across the conduction and valence bands.

We note that at low excitation fluences $\left(3.6 \mathrm{~nJ} \mathrm{~cm}{ }^{-2}\right.$, Figure 1$)$ an additional rapid initial PL decays becomes apparent towards lower temperatures, which occurs within the first few nanoseconds after photoexcitation. Such accelerated decays towards lower initial chargecarrier density cannot be explained within the approximate model described by Equation 1. In particular we note that these fast initial components are absent for higher excitation fluences $\left(280 \mathrm{~nJ} \mathrm{~cm}{ }^{-2}\right)$. As we shall show later, such fast initial decays are a signature of initial filling of trap states that terminates once traps have become saturated and therefore are barely apparent for larger densities of injected charge carriers. Thus, the presence of such fast decay 


\section{WILEY-VCH}

components requires the development of a new model that explicitly accounts for dynamic charge-carrier trapping and build-up in the material, as described in full detail further below.

\subsection{Temperature-Dependent PL Spectra and PLQE}

The shape of the time-integrated photoluminescence (PL) spectra and the quantum efficiency are important additional tools for assessing charge-carrier recombination and trapping channels in MHPs. First, these quantities allow for an assessment of whether the recombination channels observed in PL transients are radiative or non-radiative by relating these separate observations in our model, as described in Section 2.5.3. Second, the temperature dependence of the PL broadening and peak shift provides useful insights into several material properties, such as phase transition temperature and electron-phonon coupling. The phase transition temperature in particular may have an effect on charge-carrier trapping, because the accompanying changes in crystal structure may also affect other material properties such as trap energies or densities, as discussed in Section 2.5.2. ${ }^{[18]}$

Figure 2 displays false-color plots of the PL spectra of a $\mathrm{FA}_{0.95} \mathrm{Cs}_{0.05} \mathrm{PbI}_{3}$ thin film as a function of temperature for (a) high, and (b) low excitation fluences. The sudden change in PL peak shift at $150 \mathrm{~K}$ marks a phase transition, after which the PL peak energy continues to blueshift with temperature up to $295 \mathrm{~K}$. These shifts are comparable to those observed for the widely studied cesium-free $\mathrm{FAPbI}_{3}$, whose exact crystal phases and phase transition temperatures are still a matter of some debate. ${ }^{[17,19]}$ Therefore we note that changes in recombination parameters may potentially also occur at the phase transition near $150 \mathrm{~K}$. 


\section{WILEY-VCH}
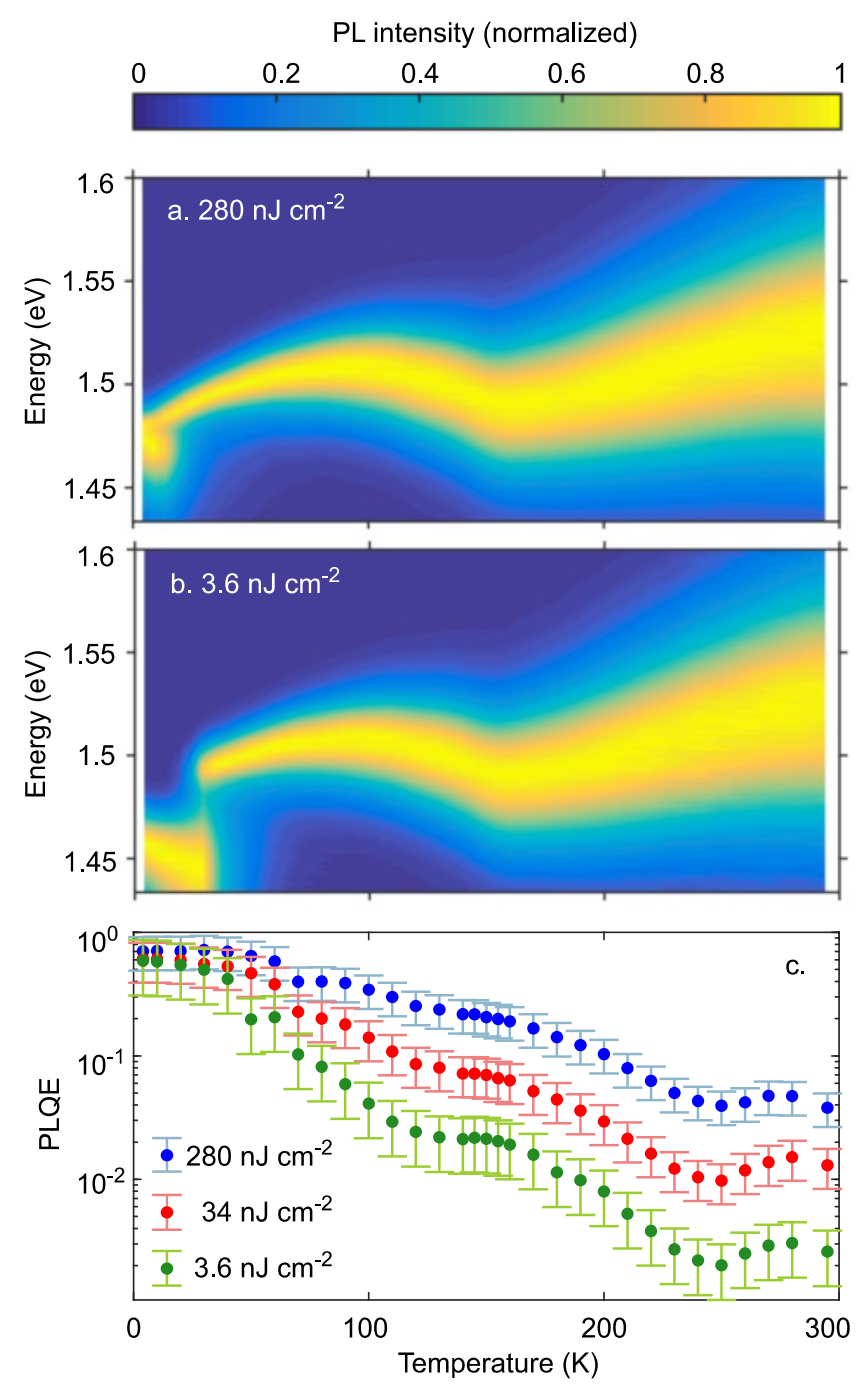

Figure 2. PL spectra and PLQE values of a $\mathrm{FA}_{0.95} \mathrm{Cs}_{0.05} \mathrm{PbI}_{3}$ thin film for temperatures between $4 \mathrm{~K}$ and $295 \mathrm{~K}$. (a) False-color plot of PL spectra at low fluence $3.6 \mathrm{~nJ} \mathrm{~cm}^{-2}$ and (b) high fluence $280 \mathrm{~nJ} \mathrm{~cm}^{-2}$ as a function of temperature. At very low temperatures band-tail PL emission dominates, showing sub-band-gap PL emission that redshifts with decreasing excitation fluence. A phase transition occurs at $150 \mathrm{~K}$. (c) PL quantum efficiency, scaled by the reference standard Rhodamine $6 \mathrm{G}$ at room temperature. Nearly all charge-carrier recombination at low temperatures is radiative and the PLQE approaches unity. With increasing temperature, the external PLQE decreases, suggesting a stronger non-radiative and/or weaker radiative band-to-band recombination process.

In addition, Figure $2 \mathrm{a}$ and $2 \mathrm{~b}$ allows us to examine changes in the lineshape of the PL

emission profile with temperature. We find that above approximately $50 \mathrm{~K}$, PL spectra are broadened independent of excitation fluence, with linewidths becoming broader towards higher temperatures. Such lineshape broadening has previously been attributed to Fröhlich coupling of charge carriers with longitudinal optical phonon modes ${ }^{[20]}$ which increases with increasing phonon mode occupancy at higher temperatures. We note that at low temperatures 


\section{WILEY-VCH}

$(\mathrm{T}<50 \mathrm{~K})$ sub-band-gap PL emission occurs that blueshifts with increasing excitation

fluence. Such radiative sub-band-gap emission has previously been reported for $\mathrm{FAPbI}_{3}$ and $\mathrm{MAPbBr}_{3}$, and has been attributed to recombination of trapped electrons in band-tail states with free charge carriers. ${ }^{[21]}$ These states have been suggested to arise from minor disorder, relating e.g. to the freezing out of the organic cation's rotational motion and the tilting of the inorganic octahedron lead-halide network. Such disorder causes an exponential distribution of trap states with varying charge-carrier lifetimes depending on trap depth. ${ }^{[21 b]}$ At the lowest excitation fluence we employed, sub-band-gap PL emission is observed for temperatures below $50 \mathrm{~K}$, whereas at the highest excitation fluence, sub-band-gap PL emission only occurs below $10 \mathrm{~K}$ because of band tail saturation with charge carriers. ${ }^{\text {[21a] }}$ Such sub-band-gap PL emission follows a complex dynamic ${ }^{[21 a]}$ resulting from carrier migration through localized states. Therefore, we will only consider the charge-carrier dynamics in the temperature regime above $50 \mathrm{~K}$, for which band-to-band recombination is shown to be the only radiative recombination channel, and non-radiative recombination is assumed to result from randomly distributed localized trap states.

To enable us ultimately to disentangle the radiative and non-radiative contributions of the charge-carrier dynamics, we also determined the external PLQE of the $\mathrm{FA}_{0.95} \mathrm{Cs}_{0.05} \mathrm{PbI}_{3}$ thin film as a function of temperature, shown in Figure 2c. To date, the nature of radiative and non-radiative recombination channels in MHP materials are still the subject of some debate, which our modelling further below will clarify. The external PLQE was determined by using Rhodamine $6 \mathrm{G}$ as a reference standard at room temperature in order to scale the PL intensities recorded at different temperatures - full details are provided in Section 5 of the Supporting Information. Figure $2 \mathrm{c}$ shows that the PLQE varies with excitation fluence from $\mathrm{PLQE}=0.3 \pm 0.2 \%$ (low fluence) to PLQE $=4 \pm 1 \%$ (high fluence) at room temperature, and tends towards fully radiative emission $(\mathrm{PLQE}=1)$ at low temperature. These trends result from 


\section{WILEY-VCH}

the aforementioned changes in the relative contribution of radiative and non-radiative recombination processes with changing temperature. While at high excitation fluence, radiative bimolecular recombination contributes more to the overall decay, thus enhancing the PLQE, lower fluences result in a prominence of non-radiative recombination that lower the PLQE. Similarly, while radiative bimolecular recombination is enhanced towards lower temperatures, trap-mediated recombination declines, ${ }^{[6,11 a]}$ leading to an overall increase in PLQE. These trends will be captured quantitatively by the model described in the next subsection.

\subsection{Trap-Mediated Recombination Model}

Above, we qualitatively discuss the time-integrated and transient PL observed for a $\mathrm{FA}_{0.95} \mathrm{Cs}_{0.05} \mathrm{PbI}_{3}$ thin film. These measurements reveal rapid initial $\mathrm{PL}$ decays at low excitation fluence (Figure 1) toward lower temperatures. We conclude that the rate equation in Equation 1 cannot describe such charge-carrier dynamics meaning that an alternative model is required for a more detailed description. In the following, we show that the PL dynamics can be better described if trap-mediated recombination is explicitly accounted for by a singlestage trap-mediated recombination mechanism that also allows for charge-carrier de-trapping and trap saturation. Furthermore, we will identify the radiative and non-radiative contributions by linking the recombination parameters inferred from PL transient decays to the radiative efficiency obtained from time-integrated PL measurements. This analysis demonstrates that at low fluences, trapping and de-trapping take an important role in describing the charge-carrier dynamics. Previous models applied to perovskites have often ignored trapping or de-trapping mechanisms ${ }^{[22]}$ or exclusively investigated high or low excitation fluences. ${ }^{[10]}$ In contrast, this work covers the dynamics above and below the fluence regime at which trap saturation is expected, and also includes Auger recombination. Furthermore, the proposed model explicitly 


\section{WILEY-VCH}

considers charge-carrier de-trapping and charge-carrier accumulation deriving from earlier excitation pulses.

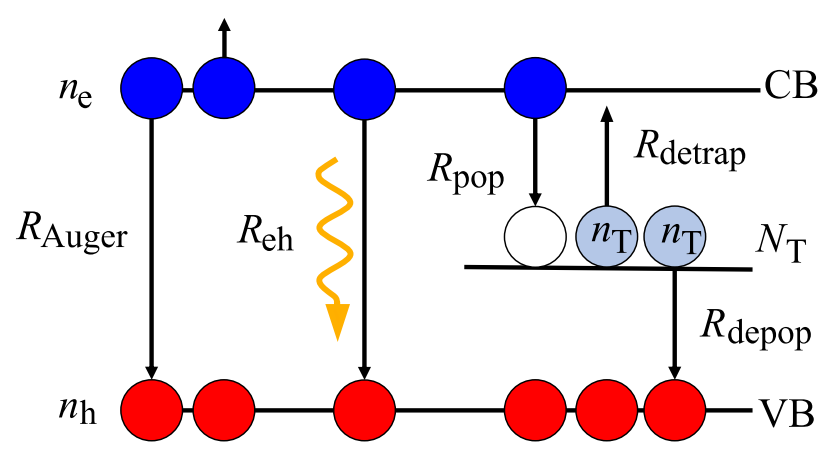

Figure 3. Schematic representation of a trap-mediated charge-carrier recombination model. Free charge-carriers with density $n_{\mathrm{e}}$ in the conduction band (CB) recombine with holes in the valence band (VB) through radiative bimolecular recombination with rate constant $R_{\text {eh }}$ or via Auger recombination with $R_{\text {Auger }}$. Furthermore, free charge carriers in the CB populate the free trap states with rate constant $R_{\text {pop. }}$ Trapped charge carriers with density $n_{\mathrm{T}}$ recombine with holes with density $n_{\mathrm{h}}$ in the VB with a rate constant $R_{\text {depop }}$; or alternatively, trapped charge carriers detrap with a rate $R_{\text {detrap }}$ to return as free charge carriers to the $\mathrm{CB}$. The total trap density is given by $N_{\mathrm{T}}$.

We proceed by deploying a single-trap-state recombination model that accounts for chargecarrier trapping and detrapping from occupied trap states, as shown schematically in Figure 3. Here, the overall charge-carrier density comprises a population of free electrons with density $n_{\mathrm{e}}$ and holes $n_{\mathrm{h}}$ in the conduction and valence band respectively, and a population of trapped electrons with density $n_{\mathrm{T}}$ in trap states energetically located within the band gap. The total trap density $N_{\mathrm{T}}$ poses an upper limit to the density of filled traps $n_{\mathrm{T}}$. Previous studies have suggested that point defects in MHPs predominantly trap electrons (rather than holes), therefore, we assume here a prominence of electron traps situated energetically below the conduction band. ${ }^{[23]}$ However, the converse situation of hole traps located above the valence band would be equally well described by the model. 


\section{WILEY-VCH}

In addition, the model illustrated in Figure 3 features the same higher-order recombination channels as the previously discussed simplified rate equation: third-order Auger

recombination described by rate constant $R_{\text {Auger }}$ and bimolecular electron-hole recombination described by rate constant $R_{\text {eh. }}$. However, trap-mediated recombination channels now also include the finite nature of trap numbers, and the ensuing possibility of trap saturation. Here, trap-mediated recombination is mediated by a single trap state involving electronic transitions between the conduction band, sub-band-gap trap states and the valence band. The trap population rate constant $R_{\text {pop }}$ governs the rate at which free electrons from the conduction band populate unoccupied trap states of density $\left(N_{\mathrm{T}}-n_{\mathrm{T}}\right)$. Once trapped, electrons may either de-trap with an associated rate constant $R_{\text {detrap }}$ back to the conduction band, or depopulate with a rate $R_{\text {depop }}$ via non-radiative recombination with a hole in the valence band.

These processes are captured in terms of the following set of rate equations,

$$
\begin{aligned}
& \frac{\mathrm{d} n_{\mathrm{e}}}{\mathrm{d} t}=-R_{\text {pop }}\left(N_{\mathrm{T}}-n_{\mathrm{T}}\right) n_{\mathrm{e}}+R_{\text {detrap }} n_{\mathrm{T}}-R_{\mathrm{eh}} n_{\mathrm{e}} n_{\mathrm{h}}-R_{\text {Auger }}\left(n_{\mathrm{e}} n_{\mathrm{h}} n_{\mathrm{h}}+n_{\mathrm{e}} n_{\mathrm{e}} n_{\mathrm{h}}\right) \\
& \frac{\mathrm{d} n_{\mathrm{T}}}{\mathrm{d} t}=+R_{\text {pop }}\left(N_{\mathrm{T}}-n_{\mathrm{T}}\right) n_{\mathrm{e}}-R_{\text {detrap }} n_{\mathrm{T}}-R_{\text {depop }} n_{\mathrm{T}} n_{\mathrm{h}}
\end{aligned}
$$

which describe the temporal evolution of free and trapped carrier densities, $n_{e}$ and $n_{\mathrm{T}}$, respectively. ${ }^{[10 a, 24]}$ We assume charge-carrier balance upon photoexcitation, i.e. the total density of electrons in conduction band and trap states has a matching hole density $n_{\mathrm{h}}$ in the valence band, meaning $n_{\mathrm{h}}=n_{\mathrm{e}}+n_{\mathrm{T}}$.

We note that excitons are not explicitly included in the model because under our experimental conditions employed, electrons and holes are excited high above the band gap using photons of energy near $3.1 \mathrm{eV}$, which will generate predominantly free charge carriers. The subsequent formation process of excitons from these free charge carriers is a bimolecular 


\section{WILEY-VCH}

process, ${ }^{[25]}$ while their radiative recombination is monomolecular. As excitonic recombination is expected to be mostly radiative, such two-step exciton formation and recombination is impossible to distinguish from the bimolecular electron-hole band-to-band recombination of free carriers. ${ }^{[10 a]}$ Therefore, the contribution of excitons to the radiative emission is essentially included in the overall bimolecular rate constant $R_{\mathrm{eh}}$. Moreover, since relatively low exciton binding energies ranging from $2-63 \mathrm{meV}$ have been reported ${ }^{[26]}$ for lead-iodide based MHPs, excitons are commonly dissociated at room temperature and potentially only play a significant role at low temperatures.

\subsection{Charge-Carrier Accumulation}

We find that one crucial aspect to ensure an accurate description of charge-carrier recombination dynamics is the inclusion of charge-carrier accumulation effects. In typical PL transient measurements, excitation occurs via a train of pulses separated in time by the inverse repetition rate, typically in the $\mathrm{MHz}$ region. However, prior to the arrival of a new pulse, a substantial fraction of charge carriers generated by the previous pulse may have remained, in particular for remnants of trapped carriers that recombine only very slowly. We note that previous studies ${ }^{[10 a, 24,27]}$ have neglected such remnant densities of trapped charges, which could cause the dynamics to depend heavily on excitation fluence and laser pulse rate.

Figure 4 demonstrates that these effects indeed require careful consideration for the $\mathrm{FA}_{0.95} \mathrm{Cs}_{0.05} \mathrm{PbI}_{3}$ thin film under investigation here. PL transients recorded at different laser pulse repetition rate but identical excitation fluence reveal that the magnitude of the initial rapid PL decay increases substantially if the repetition frequency is decreased. Increasing the time interval between excitation pulses allows more time for trap states to depopulate, meaning that a larger number of trap states will be unoccupied at the end of the cycle. As a result, newly injected free charge carriers find a higher density of vacant trap states, resulting 


\section{WILEY-VCH}

in larger initial PL decays when the laser pulse rate is lowered. Our experimental results therefore demonstrate that any initially free and trapped charge-carrier densities need to be carefully determined, if charge-carrier kinetics are to be represented accurately through a rate equation model.

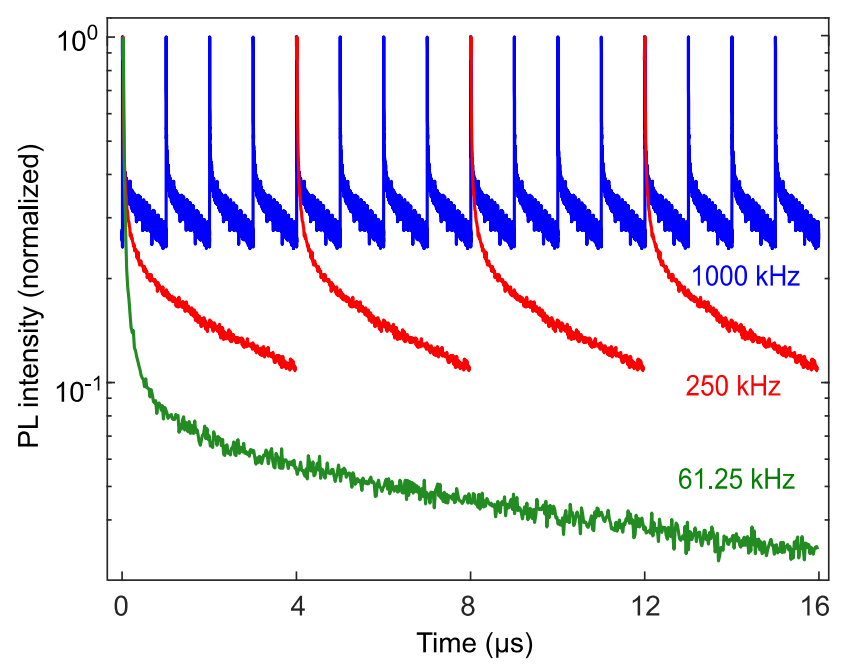

Figure 4. PL transients recorded for excitation pulse repetition rates of $1000 \mathrm{kHz}, 250 \mathrm{kHz}$ and $61.25 \mathrm{kHz}$, at a temperature of $100 \mathrm{~K}$ at a low excitation fluence of $3.6 \mathrm{~nJ} \mathrm{~cm}{ }^{-2}$ and a $398 \mathrm{~nm}$ excitation wavelength. At lower laser pulse repetition rates, trapped charge carriers have more time to depopulate, resulting in a larger number of vacant trap sites being available prior to the arrival of a subsequent laser pulse, and therefore higher initial trap-related recombination rates, compared to measurements at high repetition rates.

To account for such effects in our model, we explicitly include any accumulation of free and trapped charge-carriers. Prior to the arrival of each subsequent laser pulse, carriers will have either recombined or will remain in the model system as free or trapped charge carriers at the commencement of the next excitation cycle. We calculate free and trapped charge-carrier densities for multiple iterations, adding a newly injected charge-carrier density $n_{0}$ at the beginning of each iteration representing the arrival of a new excitation pulse. Here, the injected charge-carrier density $n_{0}$ is derived from the laser pulse fluence, as described in Section 8.1 of the Supporting Information. We performed subsequent iterations over cycles 


\section{WILEY-VCH}

incorporating remnant charge populations while adding newly injected carriers, until a final state was reached for which the charge-carrier dynamics no longer varied between consecutive cycles. A full description of this approach is provided in Section 10 of the Supporting Information. This explicit calculation of free and trapped carriers under photoexcitation ensures that the initial charge-charge carrier densities present at the beginning of each cycle are taken correctly into account.

\subsection{Model Parameters and Results}

We employ the solutions to rate equations (Equation 2 and 3) in order to replicate the PL transients across different temperatures and excitation fluences. As mentioned earlier, we restrict our analysis to temperatures above $50 \mathrm{~K}$, owing to the occurrence of band tail emission at lower temperatures. Figure 5 demonstrates for a number of sample transients that the model is able to replicate the transients with high accuracy - a full set of fits to all data is shown in Figure S13 in the SI. Because the model has to incorporate a large number of parameters, we decided to place certain reasonable restrictions on some of them in order for meaningful values to be returned for the rest. Both the assumptions, and the lessons learnt from the returned parameter values (shown in Figure 6) will be discussed in detail in this subsection. Full details on individual fitting parameters returned, and calculated inputs such as the initial charge-carrier densities, and values for the bimolecular recombination rate constant, are provided in Sections 8 of the Supporting Information. 

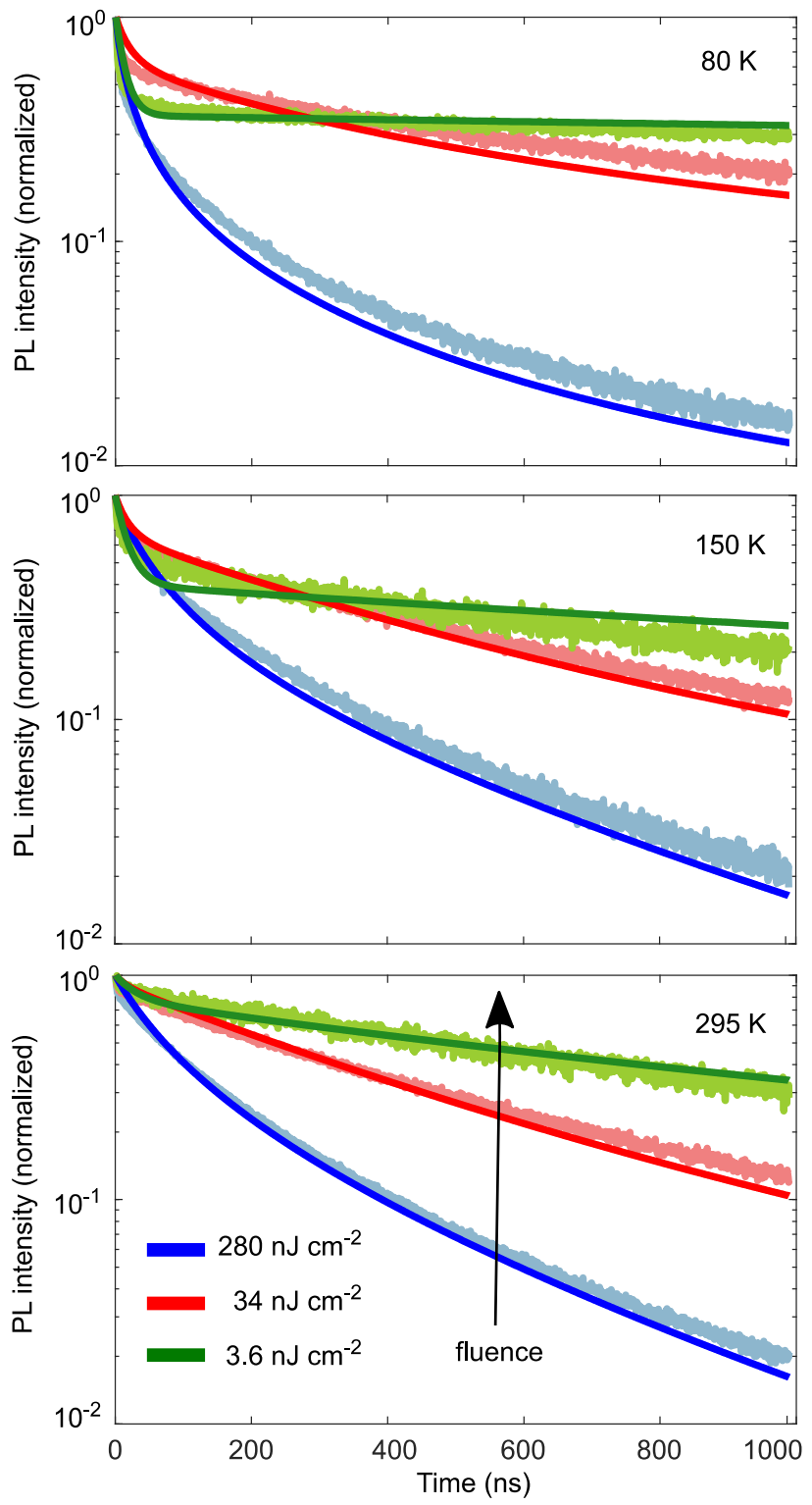

Figure 5. Measured (faded colors) and modelled (bright colors) PL transients for a $\mathrm{FA}_{0.95} \mathrm{Cs}_{0.05} \mathrm{PbI}_{3}$ thin film, at selected temperatures and across three different excitation fluences. The fits are obtained following Equation 2 and 3 as outlined in the main text. Rapid initial PL decays at low fluence result from rapid population of unoccupied trap states by photoinjected free charge carriers.

\subsubsection{Higher-order charge-carrier recombination}

In our model we include bimolecular and Auger recombination, both of which will only become prominent in the regime of high excitation fluence. To enhance accuracy in this multi-parameter space, we pre-determine the bimolecular recombination rate constant $R_{\text {eh }}$ by deriving its values from the instantaneous PL emission intensity, as previously proposed by 


\section{WILEY-VCH}

Sarritzu et al. ${ }^{[28]}$ If we assume bimolecular band-to-band recombination to be the only radiative recombination channel operating in this material at any temperature, ${ }^{[6]}$ the PL intensity must be given by $I(t) \propto R_{\mathrm{eh}} n_{\mathrm{e}}(t) n_{\mathrm{h}}(t)$ at all times, according to Equation 2 . In particular, right after photoexcitation at time $t_{0}$, the initial electron and hole density can be approximated by $n_{\mathrm{e}}\left(t_{0}\right) \approx n_{\mathrm{h}}\left(t_{0}\right) \approx n_{0}$, where $n_{0}$ is the known charge-carrier density excited per laser pulse. This approach allows us to relate the instantaneous PL intensity emitted $I_{0}$ right after laser excitation to the bimolecular recombination rate constant $I_{0}(T)=R_{\mathrm{eh}} n_{0}^{2}$ at each temperature. However, since only a fraction of the emitted light is collected, only a quantity proportional to $I_{0}$ is known. Therefore, we determine the value of the effective bimolecular recombination constant $R_{\text {eh }}$ (including effects of photon reabsorption) at room temperature for $\mathrm{FA}_{0.95} \mathrm{Cs}_{0.05} \mathrm{PbI}_{3}$ through optical-pump terahertz-probe transient photoconductivity spectroscopy to be $(1.5 \pm 0.2) \times 10^{-11} \mathrm{~cm}^{3} \mathrm{~s}^{-1}$ (full details are provided in Section 8.2 of the Supporting Information) and use it to scale the values of $R_{\text {eh }}$ obtained from the instantaneous PL across all temperatures. The resulting bimolecular recombination rate constant $R_{\text {eh }}$ is shown in the inset of Figure 6 a as a function of temperature. We find that bimolecular recombination rate constants increase for lowered temperatures, in accordance with the observed increased charge-carrier recombination rates observed experimentally at high excitation fluences (Figure 1) and reports in previous studies. ${ }^{[6,11 \mathrm{a}]}$

Having determined $R_{\text {eh }}$ independently, we introduce $R_{\text {Auger }}$ as a parameter that is obtained from fitting the model solutions to the PL transients (Figure 5) across three excitation fluences at each given temperature. The resulting Auger recombination rate constants are shown in Figure 6a as a function of temperature. Above the phase transition $(\mathrm{T}>150 \mathrm{~K})$, the Auger recombination rate constant $R_{\text {Auger }}$ is found to be roughly constant at a value of $R_{\text {Auger }}=$ $(2.1 \pm 0.5) \times 10^{-27} \mathrm{~cm}^{6} \mathrm{~s}^{-1}$. Below the phase transition, $R_{\text {Auger }}$ increases with decreasing temperature up to $R_{\text {Auger }}=(5.8 \pm 0.2) \times 10^{-27} \mathrm{~cm}^{6} \mathrm{~s}^{-1}$. A previous study on $\mathrm{MAPbI}_{3}$ thin films by 


\section{WILEY-VCH}

Milot et al. has shown a qualitatively similar dependence of the Auger recombination constant in the respective crystal phases, but reported a much larger increase of the Auger recombination constant towards lower temperatures ${ }^{[11 \mathrm{a}]}$ than we observe here. In contrast, the Auger recombination coefficient of undoped silicon and bulk indium-gallium-arsenide (InGaAs) is reported to decrease towards lower temperatures following experimental and theoretical studies. ${ }^{[29]}$ Such discrepancies between different material systems are to be expected, given that Auger recombination is highly sensitive to the electronic band structure ${ }^{[30]}$ resulting from the need for both energy and momentum conservation in the overall transition.

\subsubsection{Trap-mediated recombination}

We now proceed to analyze the parameters affecting the trapping of charge carriers, and the saturation of such trap states, processes which become most prominent for low excitation fluences. To put the threshold densities at which charge-carrier trapping typically dominate in MHPs into the context of past literature, we note that previous studies have suggested that trap-related processes saturate for free charge-carrier densities above $\approx 10^{15} \mathrm{~cm}^{-3}$, above which band-to-band recombination becomes dominant. ${ }^{[22,27]}$ Below such threshold chargecarrier densities, fast initial PL decays upon photo-excitation have been observed and attributed to rapid localization of free charge carriers in trapping centers, ${ }^{[10 \mathrm{~b}]}$ resulting in a rapid decrease of free charge carriers. In this work, we have explored charge-carrier densities ranging from $3 \times 10^{14} \mathrm{~cm}^{-3}$ to $3 \times 10^{16} \mathrm{~cm}^{-3}$ which cover the regime below and above where we expect trap saturation effects to show an onset, although we note that trap densities are expected to vary with processing routes and material systems. We extract information on detrapping rates, trap densities and trap depopulation rates from our model, as discussed in detail below. 


\section{WILEY-VCH}
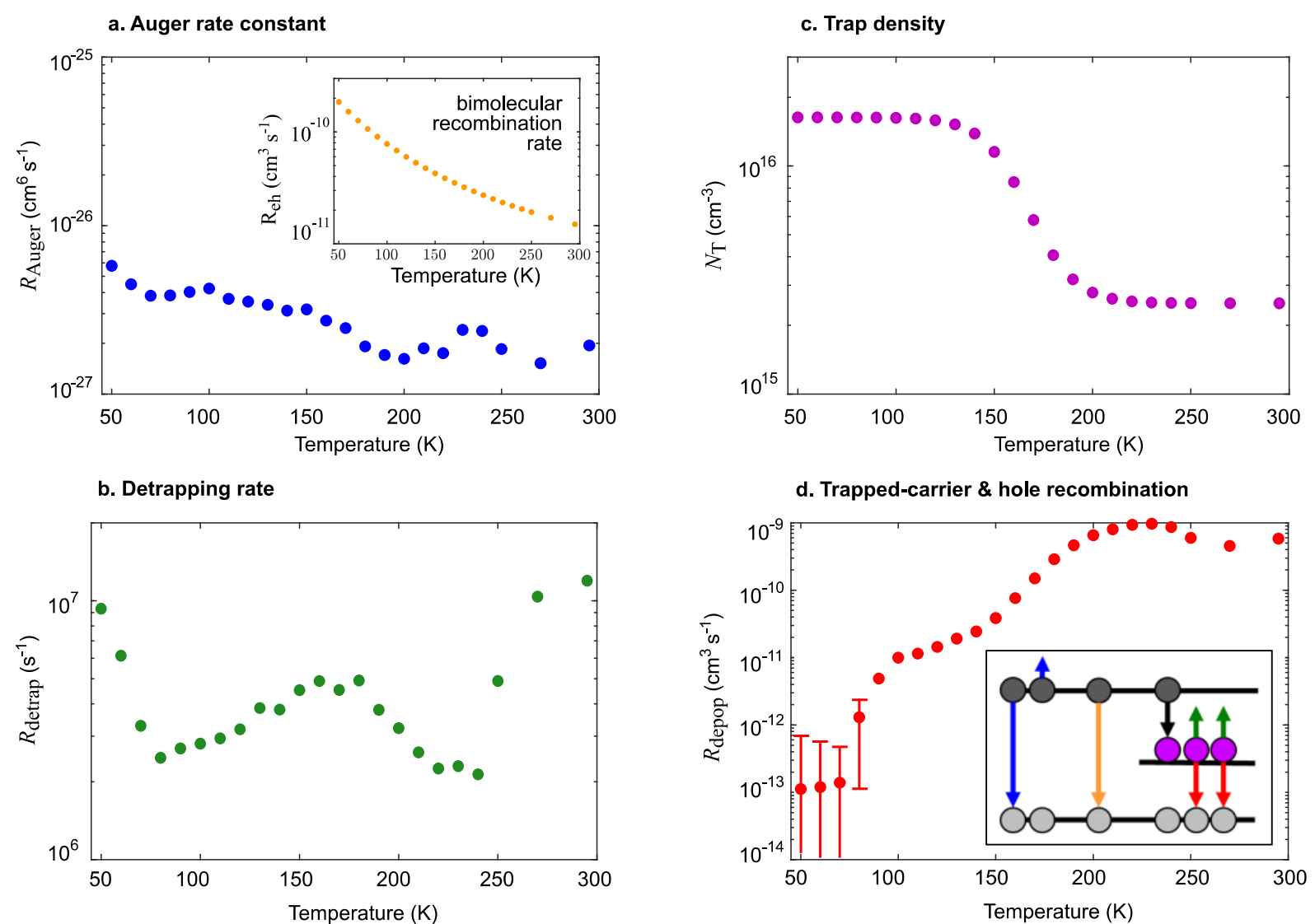

Figure 6. Parameters extracted from analysis and fits of $\mathrm{PL}$ transients for a $\mathrm{FA}_{0.95} \mathrm{Cs}_{0.05} \mathrm{PbI}_{3}$ thin film across temperatures between $50 \mathrm{~K}$ and $295 \mathrm{~K}$ and three excitation fluences. The bimolecular recombination constant $R_{\mathrm{eh}}$ (inset in a.) is determined from the instantaneous PL emission intensity, scaled by a room-temperature value extracted from $\mathrm{THz}$ photoconductivity transients. (a) The Auger recombination constant $R_{\text {Auger }}$ and (b) the de-trapping rate constant $R_{\text {detrap }}$ are extracted from global fits to transients across three excitation fluences, at each given temperature. (c) The trap density $N_{\mathrm{T}}$ is determined from global fits to transients across three fluences and all temperature, using four input parameters and assuming a temperature dependence of the form $N_{\mathrm{T}}(T)=N_{0}+\frac{\Delta N}{1+\exp \left(-b\left(T-T_{\text {shift }}\right)\right)}$. (d) The depopulation rate constant $R_{\text {depop }}$ is extracted from global fits to transients across three excitation fluences, at each given temperature and represents the final recombination of trapped electrons with VB holes. A population constant $R_{\text {pop }}=7.6 \times 10^{-9} \mathrm{~cm}^{3} / \mathrm{s}$ is returned by global fits across all transients. The schematic model in inset (d) shows the different transitions in the colors corresponding to those used for the graphical display of the corresponding individual rate constants.

First, we discuss the rate at which charge carriers may be de-trapped following their initial capture, reflected in the de-trapping rate constant $R_{\text {detrap}}$, which is optimized at each temperature globally across all three excitation fluences and shown in Figure $6 \mathrm{~b}$ as a function of temperature. The de-trapping rate exhibits two temperature ranges below and above the phase transition for which it increases continuously with temperature, in accordance with a thermally activated de-trapping mechanism. However, within a region near the phase 


\section{WILEY-VCH}

transition $(\approx 150 \mathrm{~K}$ to $250 \mathrm{~K})$ a decline in de-trapping is instead observed with rising temperature. We note that this counter-intuitive effect may arise from actual changes in the energy offsets or density of traps near the phase transition, ${ }^{[18]}$ which are discussed in more detail below. Thermal de-trapping, photon- or phonon-assisted depopulation, impact ionization and tunneling are amongst the de-trapping mechanisms that have been proposed to occur in semiconductors. ${ }^{[31]}$ Outside the phase-transition region, our findings are best explained by thermally activated de-trapping from relatively shallow trap states, ${ }^{[10 b, 11 a, 12]}$ which has been proposed before to occur in a variety of different MHPs. ${ }^{[11 a, 16 b, 31,32]}$

We note that while the aforementioned de-trapping rate constant $R_{\text {detrap }}$ is allowed to vary with temperature, we assume the trap population rate constant $R_{\text {pop }}$ to be constant, obtaining a value of $R_{\text {pop }}=7.6 \times 10^{-9} \mathrm{~cm}^{3} \mathrm{~s}^{-1}$ from global fits across all temperatures and excitation fluences. This step is taken because trap population naturally tends to be less reliant on thermal energy than trap depopulation. We are unable to separate the thermal effects on detrapping and trap population constants reliably in these fits because the model is mostly sensitive to the net trap population rate, which is given by the difference of de-trapping and trap population rates. Both rates determine the net transitions of charge-carriers between trap states and the conduction band, and occur on a faster time scale than recombination processes, making them intrinsically difficult to disentangle. Therefore, we note that it is possible that $R_{\text {pop }}$ carries a temperature dependence that is neglected here.

Our model solutions show that the PL transients are highly sensitive to the net trapping rate $\Delta R_{\text {trap }}$, which describes the effective density of charge carriers transferred to traps through $\Delta R_{\text {trap }}=R_{\text {pop }}\left(N_{\mathrm{T}}-n_{\mathrm{T}}\right) n_{\mathrm{e}}-R_{\text {detrap }} n_{\mathrm{T}}$. Initially upon pulsed excitation, the rates are out of balance because a high density of free carriers is newly excited to the conduction band, while many trap states are still unoccupied, resulting in a large net trapping rate and fast initial PL 


\section{WILEY-VCH}

decays. Within nanoseconds however, a quasi-equilibrium is reached and only a small net trapping rate remains, leading to the observed flattening of the decay dynamics. Further details on how net trapping rates affect charge-carrier dynamics can be found in Section 9.2 of the Supporting Information.

From our model fits, we are further able to obtain information on the total trap density $N_{\mathrm{T}}(T)$ present in the $\mathrm{FA}_{0.95} \mathrm{Cs}_{0.05} \mathrm{PbI}_{3}$ thin film across different temperatures. We find that our first attempt of assuming a constant trap density to be present, independent of sample temperature, was unable to provide satisfactory fits to the measured transients. As described in more detail in Section 11 of the Supporting Information, such a constant trap density cannot account correctly for the temperature-dependence of the initial rapid charge-carrier decays, in particular near the phase transition. Since previous studies had suggested that trap properties in MHPs may change near the phase transition ${ }^{[18]}$, we therefore decided to incorporate the possibility for a gradual shift in trap density $N_{\mathrm{T}}(T)$ across the phase transition region, but assumed constant values within a given crystal phase. To incorporate this change into a model, we presumed the trap density $N_{\mathrm{T}}(T)$ to assume low-temperature $\left(N_{\mathrm{T}, \mathrm{low}}=N_{0}\right)$ and high-temperature $\left(N_{\mathrm{T}, \text { high }}=N_{0}+\Delta N\right)$ values, with a gradual change in trap density centered at the temperature $T_{\text {shift }}$ according to $N_{T}(T)=N_{0}+\frac{\Delta N}{1+\exp \left(-b\left(T-T_{\text {shift }}\right)\right)}$. The temperature $T_{\text {shift }}$ at which the trap density shift occurs, and the width of the transition region, governed by $b$, as well as the minimal and maximal trap densities are allowed to vary globally across all temperatures and three excitation fluences. Figure $6 \mathrm{c}$ displays the temperature dependence of the resulting trap density obtained from such fits to PL transient data. We note that the extracted value of $T_{\text {shift }}=157 \pm 5 \mathrm{~K}$ agrees well with the temperature at which a phase transition appears to occur according to the PL spectra displayed in Figure 2 a. We also note that the trap density extracted for the high temperature phase is estimated at $2.5 \times 10^{15} \mathrm{~cm}^{-3}$ and 


\section{WILEY-VCH}

$1.5 \times 10^{16} \mathrm{~cm}^{-3}$ for the low temperature phase. Both values are close to defect densities typically estimated for similar MHPs. ${ }^{[10 \mathrm{a}, 22 \mathrm{~b}]}$ In addition, our findings suggest that the defect density appears to be fortuitously lowered for the high-temperature phase of $\mathrm{FA}_{0.95} \mathrm{Cs}_{0.05} \mathrm{PbI}_{3}$, which is positive for their use in photovoltaic applications.

Finally, the depopulation rate constant $R_{\text {depop }}$, which describes the recombination of trapped electrons with holes in the valence band, is displayed in Figure $6 \mathrm{~d}$ as a function of temperature. The depopulation rate constant $R_{\text {depop }}$ increases with temperature, in agreement with the proposed temperature dependence of SRH recombination, as previously observed in $\mathrm{MAPbI}_{3},{ }^{[11 \mathrm{a}]}$ silicon and other semiconductors. ${ }^{[16]}$

As a closing comment on trap-mediated recombination, we note that our modelling allows us also to determine the exact extent to which "remnant" electrons remain in traps at the end of an excitation cycle (and therefore the beginning of the next cycle). To illustrate this behavior, Figure $\mathbf{7 b}$ displays the fractional occupancy of traps at the point at which another photoexcitation pulse is imminent to arrive. We find that for all excitation fluences employed, and across all temperatures, a reasonable fraction of trap states clearly remains occupied, albeit not all of them. These considerations re-iterate that charge-carrier accumulation has to be carefully accounted for in such pulsed PL transient measurements at typical laser repetition rates. In addition, they also show that even at the highest fluences employed here, which correspond to injected charge-carrier densities of $3 \times 10^{16} \mathrm{~cm}^{-3}$ (see Section 8.1 in SI), full trap saturation does not occur, and such assumptions, which have often been made in the past, ${ }^{[10 \mathrm{a}, 24,27]}$ have to be reconsidered with care. Furthermore, Figure $7 \mathrm{~b}$ reveals a decreasing ratio of trap occupancy with increasing temperature, which is expected, given the observed increase in de-trapping, as well as trap depopulation through recombination. 


\section{WILEY-VCH}

\subsubsection{Radiative efficiencies}

Finally, we compare and contrast the radiative efficiencies expected from our model with values actually measured and displayed in Figure 2c. From this process, we aim to shed light on the extent to which bimolecular band-to-band recombination is a fully radiative process, given that non-radiative contributions have also been proposed. ${ }^{[33]}$ The PLQE is defined as the ratio of all radiative recombination events, over all recombination events. ${ }^{[34]}$ Here, we assume that bimolecular recombination is fully radiative and all other recombination channels are non-radiative, and validate this assumption by comparing the resulting calculated radiative efficiency with the measured external PLQE. Under these assumptions, the radiative efficiency is given by

$$
\text { PLQE }=\frac{\int_{t_{0}}^{t_{\text {end }}} R_{\text {eh }} n_{\mathrm{e}} n_{\mathrm{h}} \mathrm{d} t}{\int_{t_{0}}^{t_{\text {end }}}\left(R_{\text {eh }} n_{\mathrm{e}} n_{\mathrm{h}}+R_{\text {depop }} n_{\mathrm{T}} n_{\mathrm{h}}+R_{\text {Auger }}\left(n_{\mathrm{e}} n_{\mathrm{e}} n_{\mathrm{h}}+n_{\mathrm{e}} n_{\mathrm{h}} n_{\mathrm{h}}\right)\right) \mathrm{d} t}
$$

where $t_{0}$ and $t_{\text {end }}$ are the times just after and prior to the arrival of photoexcitation pulses and thus span the time period of a full PL decay transient. ${ }^{[34,35]}$ We note that for our case, the bimolecular recombination rate constant $R_{\mathrm{eh}}$ is the effective recombination rate constant that relates to the external PLQE, given its method of determination (see Section 8.2 in SI). ${ }^{[36]}$ Its value differs from that of the internal bimolecular recombination rate constant because of internal reabsorption events and light out-coupling. ${ }^{[33 b, 36]}$

The resulting radiative efficiency calculated from the parameters extracted from the model through fits to the PL transients is shown in Figure 7a as a function of temperature. As expected, the PLQE rises with decreasing temperature, as a result of increased radiative bimolecular, and decreased trap-related recombination. We note that these predicted trends agree well with measured PLQE values displayed in Figure 2c, showing that the assumption 


\section{WILEY-VCH}

of fully radiative bimolecular recombination is a valid approach, in agreement with previous reports, ${ }^{[6]}$ but not others, ${ }^{[33 b]}$ which have suggested a significant non-radiative bimolecular channel in related MHPs. Such differences may for example arise from different approaches to the approaches used by the model to describe data, or differences in sample preparation and quality.

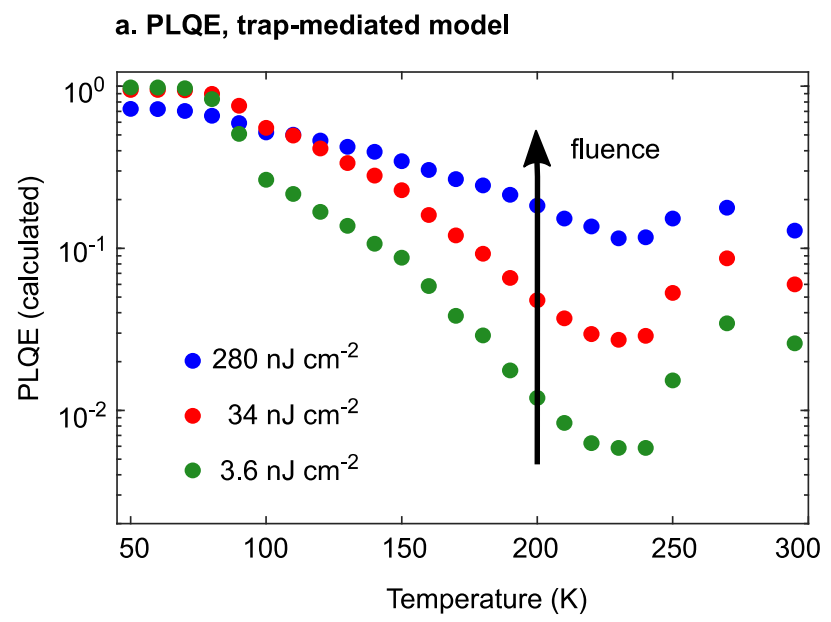

b. Remnant trap occupancy

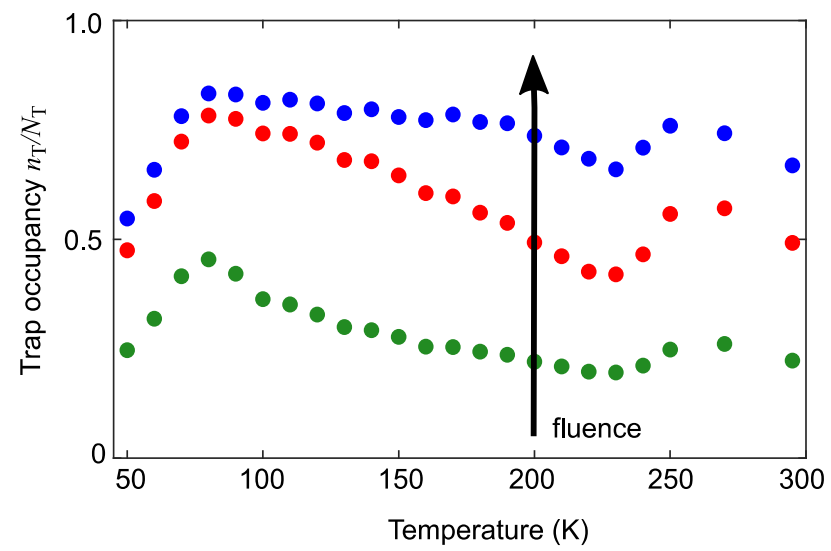

Figure 7. Expected PLQE and trap occupancy for a $\mathrm{FA}_{0.95} \mathrm{Cs}_{0.05} \mathrm{PbI}_{3}$ thin film determined from fits to transient PL based on the trapping model. (a) PLQE calculated according to Equation 4 for the three excitation fluences shown in the legend, and plotted across different temperatures. (b) Ratio of filled trap density $n_{\mathrm{T}}$ over total trap density $N_{\mathrm{T}}$ prior to laser excitation, reflecting fractional trap occupancy just before the arrival of a new laser excitation pulse, shown for three excitation fluences (according to the legend in (a)) and as a function of temperature.

\section{Conclusion}

In this study, we have presented a unified picture of charge-carrier trapping, radiative and non-radiative recombination in the prototypical metal halide perovskite $\mathrm{FA}_{0.95} \mathrm{Cs}_{0.05} \mathrm{PbI}_{3}$. We 


\section{WILEY-VCH}

find that at low excitation fluence, PL transients carry the hallmark of rapid charge-carrier trapping, exhibiting rapid decays over the first nanoseconds. A quasi-equilibrium is subsequently reached when trapping and de-trapping balance, and PL transients become mostly governed by the recombination of trapped electrons with valence-band holes over the next hundreds of nanoseconds to microseconds. For higher excitation fluences, such initial rapid trapping features are considerably weakened or visually absent because trap saturation occurs sufficiently rapidly, and the PL transients are dominated by bimolecular radiative recombination of free conduction-band electrons with free valence-band holes.

In order to describe such complex effects, we have implemented a dynamic model that accounts for charge-carrier trapping and de-trapping, trapped-charge recombination, and higher-order bimolecular and Auger recombination. Previous studies of charge-carrier dynamics in perovskite thin films have often ignored trap-saturation effects, assuming essentially a constant density of unoccupied traps to be present throughout the charge-carrier decays, ${ }^{[10 a, 24,27]}$ or else trapping and de-trapping mechanisms have been ignored ${ }^{[22]}$. In addition, the effects of charge-carrier accumulation following excitation with repeated pulses, which is almost always implemented in transient spectroscopy, has not been considered explicitly. Our model takes all of these factors into account, reproducing PL transients across a wide range of sample temperatures and excitation fluences, and predicting PLQE values that agree well with those recorded experimentally.

Our analysis clearly demonstrates that laser repetition rates have a strong influence on the initial charge trapping dynamics observed, because excitations from previous pulses create remnant charge carriers accumulating in traps. These effects are very evident in PL transients collected for different repetition rates, which show appreciably faster and more extensive initial decays when the repetition rate is lowered (i.e. the duration between excitation pulses is 


\section{WILEY-VCH}

lengthened). Such accumulation of remnant charge carriers in trap states thus causes a reduction in the initial fast rate of charge trapping that masks the actual density of traps present in the material. Our modelling further reveals that dependences of charge recombination dynamics on pulse repetition rates are prominent for low initial injected excitation densities near $10^{14}-10^{15} \mathrm{~cm}^{-3}$ which are often employed in transients PL measurements. However, even at higher densities near $10^{16} \mathrm{~cm}^{-3}$ complete trap saturation before the arrival of a new excitation pulse is not fully achieved. Therefore, if trap densities and charge dynamics are to be extracted accurately, it is essential for models to include charge-carrier accumulation effects, in particular in this charge-density regime, which encompasses conditions typically found under AM1.5 solar illumination. ${ }^{[1 \mathrm{~b}]}$

In addition, we find that the assumption of a constant density of charge traps with temperature may be inappropriate, in particular, if the temperature range explored displays structural phase transitions occurring in the material. We show that for $\mathrm{FA}_{0.95} \mathrm{Cs}_{0.05} \mathrm{PbI}_{3}$ thin films, a phase transition near $150 \mathrm{~K}$ marks a point at which the fast component associated with chargecarrier trapping changes appreciably. Fits to data based on our model reveal that this effect results from the density of traps reducing gradually around the phase transition temperature, leaving the room temperature phase of the material fortuitously with only one sixth of the traps present in the low temperature phase. The reason for these changes are unclear at this point, but may derive from subtle changes in strain, which has been associated with defects, ${ }^{[37]}$ or band alignment of defect levels. ${ }^{[18]}$ We also find that both charge-carrier detrapping, and charge-carrier recombination from trap states, are temperature activated processes, as would be expected.

Finally, we have been able to disentangle the radiative and non-radiative charge recombination channels present in $\mathrm{FA}_{0.95} \mathrm{Cs}_{0.05} \mathrm{PbI}_{3}$. Our model, upon which fits to $\mathrm{PL}$ 


\section{WILEY-VCH}

transients are based, assumes the existence of two non-radiative recombination channels, Auger and trap-mediated recombination, and one fully radiative mechanism, bi-molecular electron-hole band-to-band recombination. By tracing these processes over time and integrating to provide a measure of their relative prevalence over an excitation cycle, we are able to predict the radiative efficiency of the PL emission. The predicted PLQE shows good agreement with the experimentally determined values across temperatures between 50 $295 \mathrm{~K}$, demonstrating that bimolecular recombination is indeed a fully radiative process. PLQE values near unity are reached for temperatures below $\sim 100 \mathrm{~K}$, as a combination of both the enhancement of band-to-band recombination as the thermal spread of electrons and holes across their respective bands is suppressed, and the reduction of thermally activated trapmediated charge-carrier recombination.

Ultimately, this work presents a unified view of the radiative and non-radiative charge-carrier recombination channels, including charge-carrier trapping, de-trapping and accumulation, as well as higher order recombination mechanisms. Such considerations allow for accurate determination of these critical processes for charge-carrier densities close to those encountered under AM1.5 solar illuminations. In addition, our improved understanding of the presence and role of traps in these materials will aid the development of materials for perovskite solar cells whose efficiencies approach the Shockley-Queisser limit.

\section{Experimental Section}

Sample Preparation: $\mathrm{FA}_{0.95} \mathrm{Cs}_{0.05} \mathrm{PbI}_{3}$ thin films: $1.45 \mathrm{M} \mathrm{FA}_{0.95} \mathrm{Cs}_{0.05} \mathrm{PbI}_{3}$ solution perovskite precursor solution was prepared using a 4:1 (volume:volume) mixed solvent from anhydrous DMF (Sigma-Aldrich) and DMSO (Sigma-Aldrich). The precursor salts used were formamidinium iodide (FAI; Dyesol), cesium iodide (CsI; Alfa Aesar), lead iodide ( $\mathrm{PbI}_{2}$; TCI), and methylammonium chloride (MACl; Dyesol). The composition of FA, Cs and $\mathrm{Pb}$ 


\section{WILEY-VCH}

was mixed in solution at the molar ratios of 0.95:0.05:1.0, respectively. As a processing additive 0.05 excess of $\mathrm{MACl}$, with respect to the lead, was added to the solution. The solution was prepared in a nitrogen-filled glovebox and kept stirring overnight at room temperature. The precursor solution was spin-coated through a two-step spin coating program in a drybox with relative humidity below $20 \%$ (10 s at $1000 \mathrm{rpm}$ and $35 \mathrm{~s}$ at $6000 \mathrm{rpm})$ onto a z-cut quartz substrate. Anisole (Sigma-Aldrich) was dripped during the second step as an antisolvent, $10 \mathrm{~s}$ before the end. The films were then annealed at $100^{\circ} \mathrm{C}$ for $60 \mathrm{~min}$. Most of the volatile $\mathrm{MACl}$ was driven off during the curing process. This had a very positive influence upon the smooth appearance and optoelectronic properties of the crystalized films.

PL Spectroscopy: Samples were photoexcited above bandgap by a $398 \mathrm{~nm}$ picosecond pulsed diode laser (Picoquant PicoHarp, LDH-DC-405M). The emitted PL was coupled into a grating spectrometer (Princeton Instruments, SP-2558). The spectrally dispersed PL was directed to a photon-counting detector (PDM series from MPD) for time-resolved measurements or an iCCD (PI-MAX4, Princeton Instruments) for time-integrated PL spectral measurements. The timing of the photon-counting detector was controlled with a PicoHarp300 TCSPC event timer. Samples were measured over a temperature scale from $4 \mathrm{~K}$ to $295 \mathrm{~K}$ using a Helium cooled gas exchange cryostat (Oxford Instruments, OptistatCF2).

Unless otherwise indicated, a pulse repetition rate of $1 \mathrm{MHz}$ was used for PL measurements at fluences of $3.6 \mathrm{~nJ} \mathrm{~cm}^{-2}, 34 \mathrm{~nJ} \mathrm{~cm} \mathrm{~cm}^{-2}$ and $280 \mathrm{~nJ} \mathrm{~cm} \mathrm{~cm}^{-2}$.

PLQE: Rhodamine 6G (Sigma Aldrich, analytical standard) was dissolved in ethanol. PL intensity (as described above) and absorption spectroscopy (Fourier transform infrared spectrometer, Bruker Vertex 80v) of Rhodamine 6G solution, allowed calculation of the absolute PLQE of the perovskite films. Full details on establishing a PLQE reference can be found in Section 5 of the Supporting Information.

\section{Supporting Information}




\section{WILEY-VCH}

Supporting Information is available from the Wiley Online Library or from the author.

\section{Acknowledgements}

This work was supported by the Engineering and Physical Sciences Research Council (EPSRC) UK. M.J.T. acknowledges the European Commission for support under the Erasmus + Traineeship Programme. L.R.V.B. thanks the CDT in New and Sustainable Photovoltaics, and the Oxford-Radcliffe Scholarship, for financial support. P.M.-B. acknowledges TUM.solar in the context of the Bavarian Collaborative Research Project Solar Technologies Go Hybrid (SolTech).

Received: ((will be filled in by the editorial staff))

Revised: ((will be filled in by the editorial staff)) Published online: ((will be filled in by the editorial staff))

\section{References}

a) L. M. Herz, Annu. Rev. Phys. Chem. 2016, 67, 65; b) M. B. Johnston, L. M. Herz, Acc. Chem. Res. 2016, 49, 146; c) Y.-H. Kim, H. Cho, T.-W. Lee, Proc. Natl. Acad. Sci. 2016, 113, 11694; d) M. L. Petrus, J. Schlipf, C. Li, T. P. Gujar, N. Giesbrecht, P. MüllerBuschbaum, M. Thelakkat, T. Bein, S. Hüttner, P. Docampo, Adv. Energy Mater. 2017, 7, 1700264.

[2] National Renewable Energy Institute, Best Research-Cell Efficiency Chart, www.nrel.gov/pv/cell-efficiency.html, accessed: April 2020.

[3] a) H. J. Snaith, J. Phys. Chem. Lett. 2013, 4, 3623; b) D. Liu, T. L. Kelly, Nat.

Photonics. 2014, 8, 133; c) H. Zhou, Q. Chen, G. Li, S. Luo, T. Song, H.-S. Duan, Z. Hong, J. You, Y. Liu, Y. Yang, Science. 2014, 345, 542.

a) S. D. Stranks, ACS Energy Lett. 2017, 2, 1515; b) D. Shi, V. Adinolfi, R. Comin, M. Yuan, E. Alarousu, A. Buin, Y. Chen, S. Hoogland, A. Rothenberger, K. Katsiev, Y. Losovyj, X. Zhang, P. A. Dowben, O. F. Mohammed, E. H. Sargent, O. M. Bakr, Science. 2015, 347, 522. 


\section{WILEY-VCH}

[5] W. Shockley, H. J. Queisser, J. Appl. Phys. 1961, 32, 510.

[6] C. L. Davies, M. R. Filip, J. B. Patel, T. W. Crothers, C. Verdi, A. D. Wright, R. L.

Milot, F. Giustino, M. B. Johnston, L. M. Herz, Nat. Commun. 2018, 9, 293.

[7] a) Q. Jiang, Y. Zhao, X. Zhang, X. Yang, Y. Chen, Z. Chu, Q. Ye, X. Li, Z. Yin, J.

You, Nat. Photonics. 2019, 13, 460; b) I. L. Braly, D. W. DeQuilettes, L. M. Pazos-Outon, S.

Burke, M. E. Zier, D. S. Ginger, H. W. Hillhouse, Nat. Photonics. 2018, 12, 355.

[8] a) F. Wang, S. Bai, W. Tress, A. Hagfeldt, F. Gao, npj Flexible Electron. 2018, 2, 22;

b) W. Nie, H. Tsai, R. Asadpour, J.-C. Blancon, A. J. Neukirch, G. Gupta, J. J. Crochet, M.

Chhowalla, S. Tretiak, M. A. Alam, H.-L. Wang, A. D. Mohite, Science. 2015, 347, 522; c)

D. W. de Quilettes, S. M. Vorpahl, S. D. Stranks, H. Nagaoka, G. E. Eperon, M. E. Ziffer, H.

J. Snaith, D. S. Ginger, Science. 2015, 348, 683.

[9] a) J. M. Ball, A. Petrozza, Nat. Energy. 2016, 1, 16149; b) Z. Xiao, Y. Yan, Adv. Energy Mater. 2017, 7, 1701136.

[10] a) S. D. Stranks, V. M. Burlakov, T. Leijtens, J. M. Ball, A. Goriely, H. J. Snaith, Phys. Rev. Appl. 2014, 2, 034007; b) Y. Yamada, T. Yamada, A. Shimazaki, A. Wakamiya, Y. Kanemitsu, J. Phys. Chem. Lett. 2016, 7, 1972; c) A. Baumann, S. V, P. Rieder, M. C. Heiber, K. Tvingstedt, V. Dyakonov, J. Phys. Chem. Lett. 2015, 6, 2350.

[11] a) R. L. Milot, G. E. Eperon, H. J. Snaith, M. B. Johnston, L. M. Herz, Adv. Funct. Mater. 2015, 25, 6218; b) H. Oga, A. Saeki, Y. Ogomi, S. Hayase, S. Seki, J. Am. Chem. Soc. 2014, 136, 16948.

[12] T. Kirchartz, T. Markvart, U. Rau, D. A. Egger, J. Phys. Chem. Lett. 2018, 9, 939.

[13] a) W. Dane, S. Koch, S. Burke, R. K. Paranji, A. J. Shropshire, M. E. Zi, D. S. Ginger, ACS Energy Lett. 2016, 1, 438; b) C. Wehrenfennig, G. E. Eperon, M. B. Johnston, H. J. Snaith, L. M. Herz, Adv. Mater. 2014, 26, 1584.

[14] a) M. Saliba, T. Matsui, J. Y. Seo, K. Domanski, J. P. Correa-Baena, M. K.

Nazeeruddin, S. M. Zakeeruddin, W. Tress, A. Abate, A. Hagfeldt, M. Grätzel, Energy 


\section{WILEY-VCH}

Environ. Sci. 2016, 9, 1989; b) H. Choi, J. Jeong, H. B. Kim, S. Kim, B. Walker, G. H. Kim,

J. Y. Kim, Nano Energy. 2014, 7, 80; c) C. Yi, J. Luo, S. Meloni, A. Boziki, N. Ashari-

Astani, C. Grätzel, S. M. Zakeeruddin, U. Röthlisberger, M. Grätzel, Energy Environ. Sci.

2016, 9, 656; d) J. W. Lee, D. H. Kim, H. S. Kim, S. W. Seo, S. M. Cho, N. G. Park, $A d v$.

Energy Mater. 2015, 5, 1501310.

[15] W. Shockley, W. T. Read, Phys. Rev. 1952, 87, 835.

[16] a) A. Schenk, Solid-State Electron. 1992, 35, 1585; b) C. De Santi, M. Meneghini, M.

La Grassa, B. Galler, R. Zeisel, M. Goano, S. Dominici, M. Mandurrino, F. Bertazzi, D.

Robidas, G. Meneghesso, E. Zanoni, J. Appl. Phys. 2016, 119, 094501.

[17] a) D. H. Fabini, C. C. Stoumpos, G. Laurita, A. Kaltzoglou, A. G. Kontos, P. Falaras,

M. G. Kanatzidis, R. Seshadri, Angew. Chem., Int. Ed. 2016, 55, 15392; b) T. Chen, B. J.

Foley, C. Park, C. M. Brown, L. W. Harriger, J. Lee, J. Ru, M. Yoon, J. J. Choi, S. H. Lee, Sci. $A d v . \mathbf{2 0 1 6}, 2,10$.

[18] E. S. Parrott, R. L. Milot, T. Stergiopoulos, H. J. Snaith, M. B. Johnston, L. M. Herz, J. Phys. Chem. Lett. 2016, 7, 1321.

[19] C. L. Davies, J. Borchert, C. Q. Xia, R. L. Milot, H. Kraus, M. B. Johnston, L. M. Herz, J. Phys. Chem. Lett. 2018, 9, 4502.

[20] A. D. Wright, C. Verdi, R. L. Milot, G. E. Eperon, M. A. Perez-Osorio, H. J. Snaith, F. Giustino, M. B. Johnston, L. M. Herz, Nat. Commun. 2016, 7, 11755.

[21] a) A. D. Wright, R. L. Milot, G. E. Eperon, H. J. Snaith, M. B. Johnston, L. M. Herz, Adv. Funct. Mater. 2017, 27, 1; b) J. Fu, N. F. Jamaludin, B. Wu, M. Li, A. Solanki, Y. F. Ng, Adv. Energy Mater. 2019, 9, 1803119.

[22] a) V. D'Innocenzo, A. R. Srimath Kandada, M. De Bastiani, M. Gandini, A. Petrozza, J. Am. Chem. Soc. 2014, 136, 17730; b) Y. Yamada, T. Nakamura, M. Endo, A.Wakamiya, Y. Kanemitsu, J. Am. Chem. Soc. 2014, 136, 11610; c) Y. Yamada, M. Endo, A. Wakamiya, Y. Kanemitsu, J. Phys. Chem. Lett. 2015, 6, 482. 


\section{WILEY-VCH}

[23] a) T. Leijtens, G. E. Eperon, A. J. Barker, G. Grancini, W. Zhang, J. M. Ball, A. R. S.

Kandada, H. J. Snaith, A. Petrozza, Energy Environ. Sci. 2016, 9, 3472; b) G.-J. A. H.

Wetzelaer, M. Scheepers, A. M. Sempere, C. Momblona, J. Avila, H. J. Bolink, Adv. Mater. $\mathbf{2 0 1 5}, 27,1837$.

[24] X. Wen, Y. Feng, S. Huang, F. Huang, Y.-B. Cheng, M. Green, A. Ho-Baillie, J. Mater. Chem. C. 2016, 4, 793.

[25] a) J. Szczytko, L. Kappei, J. Berney, B. Deveaud, Phys. Rev. Lett. 2004, 93, 137401;

b) C. K. Yong, H. J. Joyce, J. Lloyd-Hughes, Q. Gao, H. H. Tan, C. Jagadish, M. B. Johnston, L. M. Herz, Small. 2012, 8, 1725.

[26] a) Y. Yamada, T. Yamada, L. Q. Phuong, N. Maruyama, H. Nishimura, A. Wakamiya, Y. Murata, Y. Kanemitsu, J. Am. Chem. Soc. 2015, 137, 10456; b) V. D'Innocenzo, G.

Grancini, M. J. P. Alcocer, A. R. S. Kandada, S. D. Stranks, M. M. Lee, G. Lanzani, H. J. Snaith, A. Petrozza, Nat. Commun. 2014, 5, 3586; c) Q. Lin, A. Armin, R. C. R. Nagiri, P. L. Burn, P. Meredith, Nat. Photonics. 2015, 9, 106; d) K. Wu, A. Bera, C. Ma, Y. Du, Y. Yang, L. Li, T. Wu, Phys. Chem. Chem. Phys. 2014, 16, 22476; e) T. J. Savenije, C. S. Ponseca, L. Kunneman, M. Abdellah, K. Zheng, Y. Tian, Q. Zhu, S. E. Canton, I. G. Scheblykin, T. Pullerits, A. Yartsev, V. Sundström, J. Phys. Chem. Lett. 2014, 5, 2189.

[27] C. Wehrenfennig, M. Liu, H. J. Snaith, M. B. Johnston, L. M. Herz, Energy Environ. Sci. 2014, 7, 2269.

[28] V. Sarritzu, N. Sestu, D. Marongiu, X. Chang, Q. Wang, S. Masi, S. Colella, A. Rizzo, A. Gocalinska, E. Pelucchi, M. L. Mercuri, F. Quochi, M. Saba, A. Mura, G. Bongiovanni, Adv. Opt. Mater. 2018, 6, 1701254.

[29] a) K. G. Svantesson, N. G. Nilsson, Phys. C: Solid State Phys. 1979, 12, 5111; b) L. Huldt, N. G. Nilsson, K. G. Svantesson, Appl. Phys. Lett. 1979, 35, 776; c) S. Hausser, G. Fuchs, A. Hangleiter, K. Streubel, W. T. Tsang, Appl. Phys. Lett. 1990, 56, 913.

[30] A. Haug, J. Phys. C: Solid State Phys. 1984, 17, 6191. 


\section{WILEY-VCH}

[31] C. K. Williams, J. Electronic Mater. 1992, 21, 711.

[32] a) G. Chen, Z. Xu, R.-j. Liao, Z. Xu, J. Appl. Phys. 2011, 110, 043724; b) K.

Schneider, K. v. Klitzing, Phys. Rev. B. 1988, 38, 6160.

[33] a) F. Staub, T. Kirchartz, K. Bittkau, U. Rau, J. Phys. Chem. Lett. 2017, 8, 5084; b) J.

M. Richter, M. Abdi-Jalebi, A. Sadhanala, M. Tabachnyk, J. P. Rivett, L. M. Pazos-Outon, K.

C. Gödel, M. Price, F. Deschler, R. H. Friend, Nat. Commun. 2016, 7, 13941.

[34] W. Tress, Adv. Energy Mater. 2017, 7, 1602358.

[35] R. L. Milot, G. E. Eperon, T. Green, H. J. Snaith, M. B. Johnston, L. M. Herz, J. Phys. Chem. Lett. 2016, 7, 4178.

[36] T. W. Crothers, R. L. Milot, J. B. Patel, E. S. Parrott, J. Schlipf, P. Müller-Buschbaum, M. B. Johnston, L. M. Herz, Nano Lett. 2017, 17, 5782.

[37] T. W. Jones, A. Osherov, M. Alsari, M. Sponseller, B. C. Duck, Y.-K. Jung, C. Settens, F. Niroui, R. Brenes, C. V. Stan, Y. Li, M. Abdi-Jalebi, N. Tamura, J. E. Macdonald, M. Burghammer, R. H. Friend, V. Bulovic, B. Bulovic, A. Walsh, G. J. Wilson, S. Lilliu, S. D. Stranks, Energy Environ. Sci. 2019, 12, 596. 
Keyword metal halide perovskites

Michael Johann Trimpl, Adam D. Wright, Kelly Schutt, Leonardo R.V. Buizza, Zhiping Wang, Michael B. Johnston, Henry J. Snaith, Peter Müller-Buschbaum, Laura M. Herz*

Title: Charge-Carrier Trapping and Radiative Recombination in Metal Halide Perovskite Semiconductors

The properties of trap states that limit the performance of hybrid perovskite solar cells and lightemitting devices are still under much debate. Herein, a unified model is presented, that accurately describes trap-related and higher-order charge-carrier recombination. This work reveals the importance of explicit accounting for charge-carrier trapping, detrapping and accumulation, and disentangles radiative and non-radiative recombination channels.

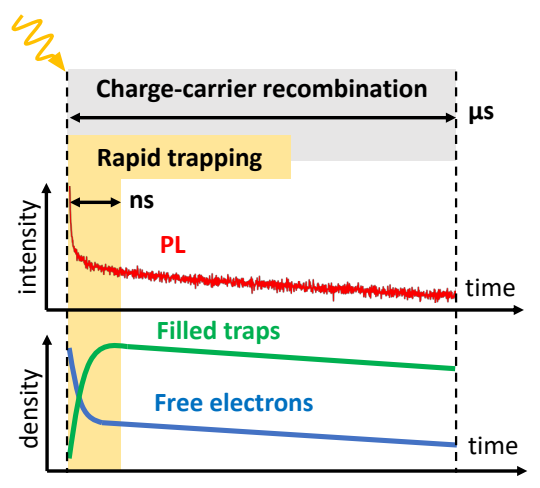




\section{WILEY-VCH}

Copyright WILEY-VCH Verlag GmbH \& Co. KGaA, 69469 Weinheim, Germany, 2018.

Supporting Information

(included as separate PDF file) 